\title{
原著 4
}

\section{Interaction of zotepine and chlorprothixene with URAT1-mediated urate transport}

\author{
Nobuyuki Onizawa ${ }^{1,2)}$ \\ Tatou Iseki ${ }^{1)}$ \\ Takayuki Hori ${ }^{1)}$ \\ Naoyuki Otani ${ }^{1)}$ \\ Motoshi Ouchi ${ }^{1)}$ \\ Keitaro Hayashi ${ }^{1)}$ \\ Hajime Hasegawa ${ }^{2)}$ \\ Naohiko Anzai ${ }^{1)}$
}

\begin{abstract}
Zotepine and chlorprothixene are antipsychotic drugs, and they have a broad therapeutic effect in patients with schizophrenia. Zotepine and chlorprothixene have been reported to decrease serum urate levels. In addition, an increase in renal clearance of urate was shown in several studies. However, the molecular mechanism underlying these effects has not yet been clarified. In the present study, we measured $\left[{ }^{14} \mathrm{C}\right]$ urate uptake using stable cells expressing human urate transporter 1 (HEK-hURAT1) and mock cells (HEK-mock) to evaluate the uricosuric actions of zotepine and chlorprothixene. Zotepine and chlorprothixene inhibited URAT1-mediated $\left[{ }^{14} \mathrm{C}\right]$ urate uptake in a concentration-dependent manner $\left(\mathrm{IC}_{50}\right.$ values were $14.52 \mu \mathrm{M}$ for zotepine and $6.08 \mu \mathrm{M}$ for chlorprothixene). Next, we measured the activity of xanthine oxidase $(\mathrm{XO})$ to determine whether zotepine and chlorprothixene have inhibitory effects on urate production. Urate production mediated by $\mathrm{XO}$ was not inhibited. Taken together, the results suggest that URAT1, a major contributor of renal urate reabsorption and a major target of uricosuric drugs such as losartan and benzbromarone, interacted with zotepine and chlorprothixene; however, $\mathrm{XO}$, a major
\end{abstract}

enzyme in urate production, did not interact with zotepine or chlorprothixene. This is the first study showing that drugs that have the structure of a tricyclic compound interact with URAT1. Elucidation of these interactions may contribute to the development of new anti-hyperuricemic agents by clarifying the structure of the substrate-binding site of URAT1.

\section{Introduction}

In humans, urate (uric acid) is the end-product of purine metabolism as a result of genetic loss of uricase $^{1,2}$. Generation of urate occurs primarily in the liver, while the excretion of urate is mainly from the kidney. Therefore, hyperuricemia results from the overproduction of urate and/or from insufficient renal elimination ${ }^{3)}$. An elevated serum urate level plays a pivotal role not only in gout but also lifestyle-related diseases, including hypertension, insulin resistance, diabetes, chronic renal disease, diabetic renal disease, and cardiovascular disease $\mathrm{e}^{4,5)}$. Thus, urate-lowering therapy is necessary for successful long-term hyperuricemia management to achieve a sustained reduction in urate, and common therapeutic targets are urate transporters in the kidney and the production

Received August 5,2015, Accepted October 12,2015

1 ) Department of Pharmacology and Toxicology, Dokkyo Medical University School of Medicine

2 ) Department of Nephrology and Hypertension, Saitama Medical Center, Saitama Medical University Key words : Urate, Zotepine, Chlorprothixene, URAT1, Xanthine Oxidase Corresponding author : Naohiko Anzai, Department of Pharmacology and Toxicology, Dokkyo Medical University School of Medicine, 880, Kitakobayashi, Mibu, Shimotsuga, Tochigi 321-0293 
pathways of urate in the liver ${ }^{6}$.

In addition to anti-hyperuricemic agents, drugs that lower serum urate levels such as losartan and salicylic acid have also been reported ${ }^{7}$. Zotepine and chlorprothixene are antipsychotic drugs that have a broad therapeutic effect in patients with schizophrenia ${ }^{8-10)}$. Table 1 illustrates the similar chemical structures of zotepine and chlorprothixene. They also have been reported to decrease serum urate levels in several clinical studies ${ }^{11-16)}$. The oral administration of $40 \mathrm{mg}$ of zotepine reduced serum uric acid levels by a mean of $2.71 \mathrm{mg} / \mathrm{dL}^{12)}$, and the oral administration of a mean of $272.7 \mathrm{mg}$ of chlorprothixene reduced serum uric acid levels by 2.64 $\mathrm{mg} / \mathrm{dL}^{14)}$. In addition, zotepine and chlorprothixene have been reported to increase the renal clearance of urate $^{11,14)}$. However, the molecular mechanism(s) of these effects remains unknown. Previous studies showed that many drugs such as probenecid, benzbromarone, and losartan can decrease the serum urate level by their inhibitory effects on URAT $1^{7}$. Human serum urate levels are mostly determined by urate reabsorption and secretion in the kidney. Renal urate reabsorption is mainly controlled via the proximal tubular urate transporter URAT 1 $(\mathrm{SLC} 22 \mathrm{~A} 12)^{7}$. Therefore, we examined the effects of zotepine and chlorprothixene on urate transporters involved in the regulation of the serum urate level.

The rate-limiting step of urate production is an enzymatic reaction of the xanthine dehydrogenase/ xanthine oxidase $(\mathrm{XDH} / \mathrm{XO})$ enzyme that oxidizes hypoxanthine-xanthine into urate. Human $\mathrm{XDH} / \mathrm{XO}$ is expressed in the liver and small intestine of $\mathrm{XDH} /$ $\mathrm{XO}$-rich parenchyma cells, and the urate production rate is based on the amount of substrate and/or XO activity ${ }^{17}$. However, the effects on urate production of zotepine and chlorprothixene have not been examined clinically. Consequently, we determined the XO inhibitory effects of these two antipsychotic drugs that reflect urate production.

\section{Methods}

\section{Materials}

$\left[{ }^{14} \mathrm{C}\right]$ urate $(57.8 \mathrm{mCi} / \mathrm{mmol})$ was purchased from Moravek Biochemicals (Brea, CA, USA). Zotepine, chlorprothixene, and benzbromarone were obtained

Table 1 Structures and molecular weights of zotepine and chlorprothixene

zotepine Structure


from Sigma Aldrich (St Louis, MO, USA). Allopurinol was obtained from Wako Pure Chemical Industries (Osaka, Japan). Fetal bovine serum was obtained from Biowest (Nuaillé, France), and Dulbecco's modified eagle's medium (DMEM), trypsin, and geneticin were purchased from Sigma Aldrich.

\section{Cell culture}

HEK-hURAT1 cells were established as previously described ${ }^{18)}$. The cells were grown in a humidified incubator at $37^{\circ} \mathrm{C}$ and in $5 \% \mathrm{CO}_{2}$ using minimum essential medium containing $10 \%$ fetal bovine serum and $500 \mu \mathrm{g} / \mathrm{mL}$ of geneticin.

\section{Urate transport activity assay}

The kinetic behavior of urate transport was examined by performing uptake studies using confluent HEKhURAT1 and HEK-mock cells. Both cell lines were cultured at a density of $1.5 \times 10^{5}$ cells/well on a 24-well plate coated with poly-D-lysine (Corning, NY, USA) for 48 hours before the uptake studies were performed. Immediately before the experiment, the medium was replaced by the uptake solution, which was chloride-free Hanks' balanced salt solution (HBSS) containing the following in mM: $125 \mathrm{Na}$ gluconate, $4.8 \mathrm{~K}$ gluconate, $1.2 \mathrm{KH}_{2} \mathrm{PO}_{4}, 1.2 \mathrm{MgSO}_{4}$, $1.3 \mathrm{Ca}$ gluconate, 5.6 glucose, and 25 HEPES, pH 7.4. After pre-incubation in the same solution in a water bath at $37^{\circ} \mathrm{C}$ for $10 \mathrm{~min}$, the cells were incubated in a solution containing $5 \mu \mathrm{mol} / \mathrm{L}$ of $\left[{ }^{14} \mathrm{C}\right]$ urate at $37^{\circ} \mathrm{C}$ for the indicated time. After 2 minutes, uptake was stopped by washing two times with ice-cold incubation medium. The cells in each well were lysed with $0.5 \mathrm{~mL}$ of $0.1 \mathrm{~N}$ sodium hydroxide and $2.5 \mathrm{~mL}$ of the liquid scintillation cocktail Insta-Gel Plus (Perkin Elmer, Waltham, MA, USA), and radioactivity was determined using a $\beta$-scintillation counter, LSC-7200 (Hitachi Aloka Medical, Tokyo, Japan). Uptake measured in HEK-mock cells was subtracted from the corresponding uptake measured in HEK-hURAT1 cells to calculate cDNA-specific uptake, after it had been calibrated using the average protein content of other wells in the same 24-well plate as that used in the uptake study, with the BCA protein assay reagent (Pierce, Rockford, IL, USA). To evaluate the inhibitory effects of zotepine, chlorprothixene, and benzbromarone on urate uptake via URAT1, HEKhURAT 1 cells were incubated in a solution containing $5 \mu \mathrm{mol} / \mathrm{L}\left[{ }^{14} \mathrm{C}\right]$ urate in the absence or presence of various concentrations $(0.01,0.1,1,3,10,30$, and $100 \mu \mathrm{M})$ of drugs at $37^{\circ} \mathrm{C}$ for $2 \mathrm{~min}$. Drugs were dissolved in dimethylsulfoxide (Wako Pure Chemical Industries). The final concentration of dimethylsulfoxide in the incubation medium was adjusted to less than $0.5 \%$.

\section{Xanthine oxidase activity assay}

We measured the activity of human XO to determine whether zotepine and chlorprothixene have inhibitory effects on urate production. In the presence of 1,10 , 50,100 , and $300 \mu \mathrm{M}$ zotepine or chlorprothixene, the fluorescence of resorufin, which is produced during uric metabolism by $\mathrm{XO}$, was analyzed using the Xanthine oxidase fluorometric assay kit (Cayman Chemical, Ann Arbor, MI, USA) according to the instructions of the manufacturer and was normalized to the protein concentration. $\mathrm{XO}$ produces $\mathrm{H}_{2} \mathrm{O}_{2}$ during the oxidation of hypoxanthine. In the presence of horseradish peroxidase in the product, $\mathrm{H}_{2} \mathrm{O}_{2}$ reacts with ADHP (10-acetyl-3,7-dihydroxyphenoxazine) in a 1:1 stoichiometry to produce the highly fluorescent compound resorufin, which can be easily analyzed ${ }^{199}$.

\section{Statistical analyses}

Uptake experiments were conducted three times, and each uptake experiment was performed in triplicate. All of the data are shown as means \pm S.E.M. Statistical differences were determined using one-way ANOVA with Dunnett' s post-hoc test. A value of $\mathrm{P}<0.05$ was considered to be significant. 


\section{Results}

\section{Urate transport activity assay}

To investigate the interaction of zotepine and chlorprothixene with URAT1, the uptake of $\left[{ }^{14} \mathrm{C}\right]$ urate was measured in chloride-free HBSS in the presence of zotepine or chlorprothixene $(50 \mu \mathrm{M})$ or in the presence of benzbromarone $(50 \mu \mathrm{M})$, which has long been used as a uricosuric drug. As shown in Fig. 1, zotepine and chlorprothixene inhibited URAT1mediated urate transport $(45 \pm 3 \%$ for zotepine and 35 $\pm 3 \%$ for chlorprothixene, respectively; $\mathrm{P}<0.01)$. We then checked the concentration dependency of these interactions. Fig. 2 shows the dependence of inhibition on the zotepine (A) and chlorprothixene (B) concentrations. The percentage of urate uptake relative to the control (no inhibitor) level decreased dosedependently from 0.01 to $100 \mu \mathrm{M}\left(\mathrm{IC}_{50}\right.$ values of $14.52 \mu \mathrm{M}$ for zotepine and $6.08 \mu \mathrm{M}$ for chlorprothixene). These findings suggest that these drugs have inhibitory action against URAT1.

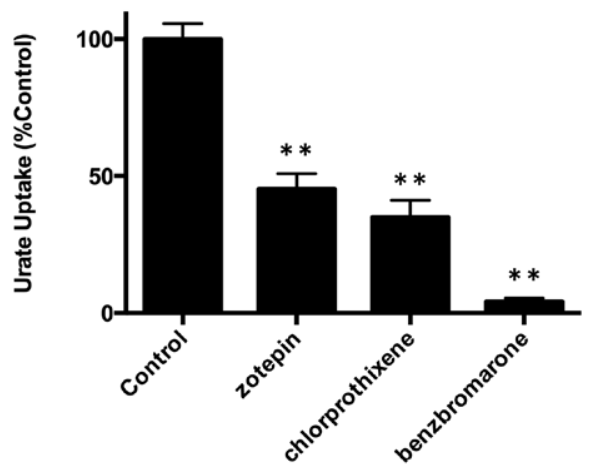

Fig. 1 Inhibition of human URAT1-mediated uric acid uptake by zotepine and chlorprothixene. Human URAT1 cDNA was expressed in HEK293 (HEK-hURAT1) cells. HEK293 cells transfected with pcDNA3.1 lacking an insert (HEK-mock) were used to determine endogenous transport. The uptake of $\left[{ }^{14} \mathrm{C}\right]$ uric acid $(5 \mu \mathrm{M})$ was measured in chloride-free Hanks' balanced salt solution (HBSS) in the presence of zotepine, chlorprothixene $(50 \mu \mathrm{M})$, or benzbromarone $(50 \mu \mathrm{M})$. The uptake measured in HEK-mock cells was subtracted from the corresponding uptake measured in cells transfected with cDNA to calculate cDNA-specific uptake. The results are expressed as a percentage of the control uptake $(100 \%)$ measured in the absence of drugs. The degree of statistical significance for uric acid uptake measured in the presence of zotepine and chlorprothixene compared to uric acid uptake measured in the absence of the drugs in hURAT1 cDNAtransfected cells is indicated by $* * \mathrm{P}<0.01$.

A

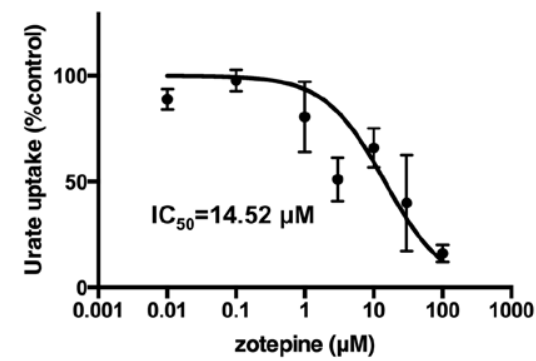

B

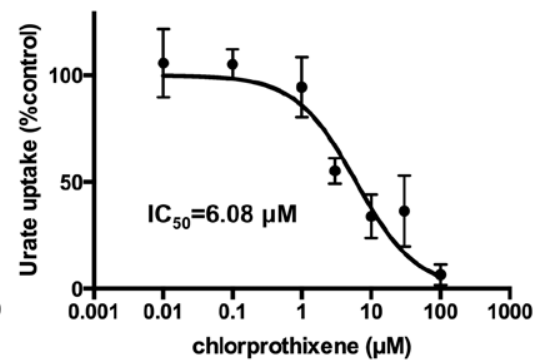

Fig. 2 Concentration-dependent inhibition of urate uptake via URAT1 by zotepine/chlorprothixene $(0.01-100 \mu \mathrm{M})$. URAT1-mediated uptake is expressed as a percentage of the no-inhibitor control (absence of drugs). The uptake of $\left[{ }^{14} \mathrm{C}\right]$ uric acid $(5 \mu \mathrm{M})$ was measured in the presence of increasing concentrations of zotepine (A) or chlorprothixene (B). The uptake measured in HEK-mock cells was subtracted from the corresponding uptake measured in cells transfected with cDNA to calculate cDNA-specific uptake. The results are expressed as a percentage of the control uptake $(100 \%)$ measured in the absence of drugs. The $50 \%$ inhibitory concentration $\left(\mathrm{IC}_{50}\right)$ values were generated using a sigmoidal dose-response relationship. 


\section{Xanthine oxidase activity assay}

The fluorescence of resorufin, which is produced during uric metabolism by $\mathrm{XO}$, was analyzed in the presence of $50 \mu \mathrm{M}$ of zotepine and chlorprothixene and was normalized to the protein concentration (Fig. 3). Allopurinol (10 $\mu \mathrm{M})$, which has long been used as an XO inhibitor, was also examined for comparison with the inhibitory effects of zotepine and chlorprothixene. Compared with the control, $50 \mu \mathrm{M}$ of zotepine and chlorprothixene did not significantly inhibit XO activity. Further inhibition study showed no concentration dependence (Fig. 4).

\section{Discussion}

To elucidate the mechanism of the urate-lowering effects of the two anti-psychotic drugs zotepine and chlorprothixene, the urate transport activity assays and the XO activity assay were conducted. In the uptake study, zotepine and chlorprothixene inhibited urate transport via URAT1 in a concentration-dependent manner. Since no XO inhibitory activity was observed in the XO activity assay, the urate-lowering effects of these drugs seem to be largely due to URAT1 inhibition.

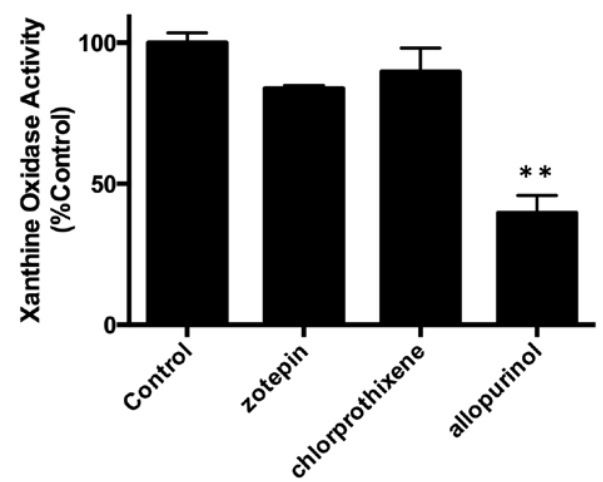

Fig. 3 Effects of zotepine and chlorprothixene $(50 \mu \mathrm{M})$ on xanthine oxidase $(\mathrm{XO})$ activity.

The fluorescence of resorufin, which is produced during uric metabolism by XO, was analyzed In the presence of $50 \mu \mathrm{M}$ of zotepine/chlorprothixene. Allopurinol $(10 \mu \mathrm{M})$ was also examined for comparison with the XO inhibitory effect of zotepine/ chlorprothixene. The results are expressed as a percentage of the control activity $(100 \%)$ measured in the absence of the drugs. No significant difference was observed between XO activity measured in the presence of zotepine/chlorprothixene and in the absence of the drugs. The degree of significance in the presence of allopurinol is indicated by $* * \mathrm{P}<0.01$.

A

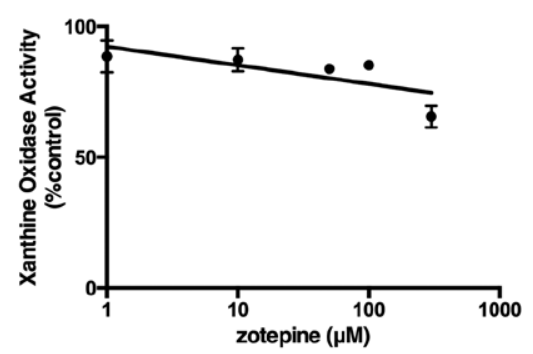

B

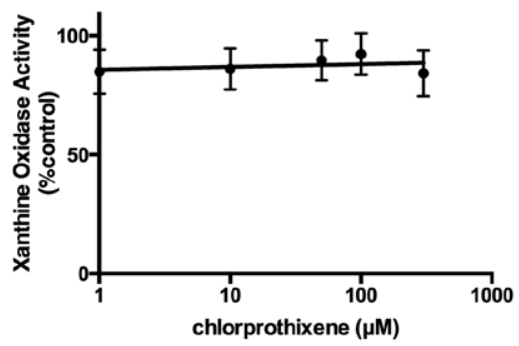

Fig. 4 Effects of zotepine and chlorprothixene (1-300 $\mu \mathrm{M}$ ) on xanthine oxidase $(\mathrm{XO})$ activity.

The fluorescence of resorufin was measured in the presence of increasing concentrations of zotepine (A) or chlorprothixene (B). The results are expressed as a percentage of the control activity (100\%) measured in the absence of the drugs. No concentration dependence was observed. 
In addition to benzbromarone, the angiotensin II receptor blocker (ARB) losartan and salicylic acid have been reported to be URAT1 inhibitors. However, there has been no study on the urate-lowering effects of drugs that have the structure of a tricyclic compound such as zotepine and chlorprothixene ${ }^{20)}$. As shown in Fig. 2, the inhibitory effects of zotepine and chlorprothixene on urate transport via URAT1 were concentration-dependent, and $\mathrm{IC}_{50} \mathrm{~S}$ of zotepine and chlorprothixene were 14.52 and $6.08 \mu \mathrm{M}$, respectively. The maximum plasma concentration ( $\mathrm{Cmax}$ ) of zotepine after oral administration $(50 \mathrm{mg})$ was approximately $0.02 \mu \mathrm{M}$ (interview form), and the serum concentration of chlorprothixene was 0.04-0.22 $\mu \mathrm{M}$ after oral administration $(100 \mathrm{mg})^{21}$. Considering that the protein-binding rates of both drugs are over $95 \%$, it is possible that the intratubular concentrations of these drugs in humans are much lower than the IC50s. Thus, the inhibition of renal uric acid reabsorption from the luminal side seems to be unlikely at the therapeutically relevant plasma concentrations of these drugs.

However, we revealed that human sodium phosphate transporter 4 (hNPT4/SLC17A3) is a multispecific organic anion efflux transporter at the apical side of renal tubules, indicating the possibility that drugs, such as loop diuretics, can be excreted via this secretory pathway ${ }^{22}$. The amount of a drug excreted into the urine via a secretory pathway, such as NPT4, may be correlated with the urate-lowering effect of the drug. Besides, the drug concentration most likely increases in the tubules owing to the volume reduction after water reabsorption. In the present study, we could not examine the interaction of these drugs because there are currently no commercially available metabolites. In addition, benzbromarone, which has long been used as a uricosuric drug, is thought to inhibit urate reabsorption from the apical side of the proximal tubule; however, benzbromarone itself is not excreted into urine. Thus, it is predicted that 6hydroxybenzbromarone, a major hydroxylated metabolite of benzbromarone, inhibits urate reabsorption mediated by URAT ${ }^{20)}$. Furthermore, drugs such as chlorpromazine that have structures similar to those of zotepine and chlorprothixene are not involved in the regulation of serum urate levels. By focusing on the effects of similar substances including their metabolites, it may be possible to identify the important structure(s) associated with URAT1.

In summary, we found that zotepine and chlorprothixene have inhibitory effects on urate transport via URAT1. Although IC50s of these two drugs are higher than their plasma concentrations, this is the first study showing that drugs that have the structure of a tricyclic compound interact with URAT1. Our findings may contribute to the development of new anti-hyperuricemic agents by clarifying the structure of the substrate-binding site of URAT1.

\section{Acknowledgments}

We thank Ms. Mio Maekawa and Mrs. Sawako Nakadate for technical assistance.

\section{Financial Disclosure}

The authors declare no conflict of interest associated with this manuscript.

\section{References}

1) Sica DA, Schoolwerth AC : Renal handling of organic anions and cations. The Kidney, edn 6, Brenner BM, WB Saunders, pp 680-700, 2000.

2) $\mathrm{Wu} X W$, Muzny DM, Lee CC et al : Two independent mutational events in the loss of urate oxidase during hominoid evolution. J Mol Evol 34 : 78-84, 1992.

3 ) Anzai N, Endou H : Drug discovery for 
hyperuricemia. Expert Opin Drug Discov 2 : 1251-1261, 2007.

4 ) Saag KG, Choi H : Epidemiology, risk factors, and lifestyle modifications for gout. Arthritis Res Ther $8:$ S2, 2006.

5 ) Anzai N, Jutabha P, Endou H : Renal solute transporters and their relevance to serum urate disorder. Curr Hypertens Rev 6 : 148-154, 2010.

6 ) Stamp LK, Chapman PT : Urate-lowering therapy: current options and future prospects for elderly patients with gout. Drugs Aging 31 : 777786, 2014.

7 ) Enomoto A, Kimura H, Chairoungdua A et al : Molecular identification of a renal urate-anion exchanger that regulates blood urate levels. Nature 417 : 447-452, 2002.

8 ) Chan H-Y, Jou S-H, Juang Y-Y et al : A singleblind, comparative study of zotepine versus haloperidol in combination with a mood stabilizer for patients with moderate-to-severe mania. Psychiatry Clin Neurosci 64 : 162-169, 2010.

9 ) Harada T, Otsuki S : Antimanic effect of zotepine. Clin Ther 8 : 406-414, 1986.

10) Fux M, Belmaker RH : A controlled comparative study of chlorprothixene vs. haloperidol in chronic schizophrenia. Isr J Psychiatry Relat Sci $28: 37-40,1991$.

11) Yoshida $H$, Kaiya $H$, Kondo $T$ et al : Effects of zotepine, a psychotropic agent, on the serum uric acid level of inpatients at a psychiatric hospital. Yakubutsu Seishin Kodo 2 : 69-76, 1982.

12) Yamamoto $T$, Maeda J, Moriwaki $Y$ et al : The effect of zotepine on the excretion of purine bases. Uric Acid Res 1: 61-68, 1985.

13) Shalev A, Hermesh H, Munitz $\mathrm{H}$ et al : Chlorprothixene-induced hypouricemia: a biologic indicator of drug compliance. J Clin
Psychiatry $50:$ 424-427, 1989.

14) Shalev A, Hermesh H, Munitz H : The hypouricemic effect of chlorprothixene. Clin Pharmacol Ther 42 : 562-566, 1987.

15) Weinshilboum RM, Goldstein JL, Kelley WN : Prolonged hypouricemia associated with acute chlorprothixene ingestion. Arthritis Rheum 18 : 739-741, 1975.

16) Healey LA, Harrison M, Decker JL : Uricosuric effect of chlorprothixene. N Engl J Med 272 : 526-527, 1965.

17) Pritsos CA : Cellular distribution, metabolism and regulation of the xanthine oxidoreductase enzyme system. Chem Biol Interact 129: 195-208, 2000.

18) Anzai N, Miyazaki H, Noshiro R et al : The multivalent $\mathrm{PDZ}$ domain-containing protein PDZK1 regulates transport activity of renal urateanion exchanger URAT1 via Its C terminus. J Biol Chem 279 : 45942-45950, 2004.

19) Amundson DM, Zhou M : Fluorometric method for the enzymatic determination of cholesterol. J Biochem Biophys Methods 38 : 43-52, 1999.

20) Shin HJ, Takeda M, Enomoto A et al : Interactions of urate transporter URAT1 in human kidney with uricosuric drugs. Nephrology (Carlton) 16 : 156-162, 2011.

21) Bagli M, Rao ML, Höflich G et al : Pharmacokinetics of chlorprothixene after single intravenous and oral administration of three galenic preparations. Arzneimittelforschung 46 : 247-250, 1996.

22) Jutabha $P$, Anzai $N$, Kitamura $K$ et al : Human sodium phosphate transporter 4 (hNPT4/ SLC17A3) as a common renal secretory pathway for drugs and urate. J Biol Chem 285 : 3512335132, 2010. 